\title{
"Planeando las compras en PartesYa": una actividad lúdica para estudiar el MRP
}

"Planeando las compras en PartesYa": a hands-on activity to study the MRP

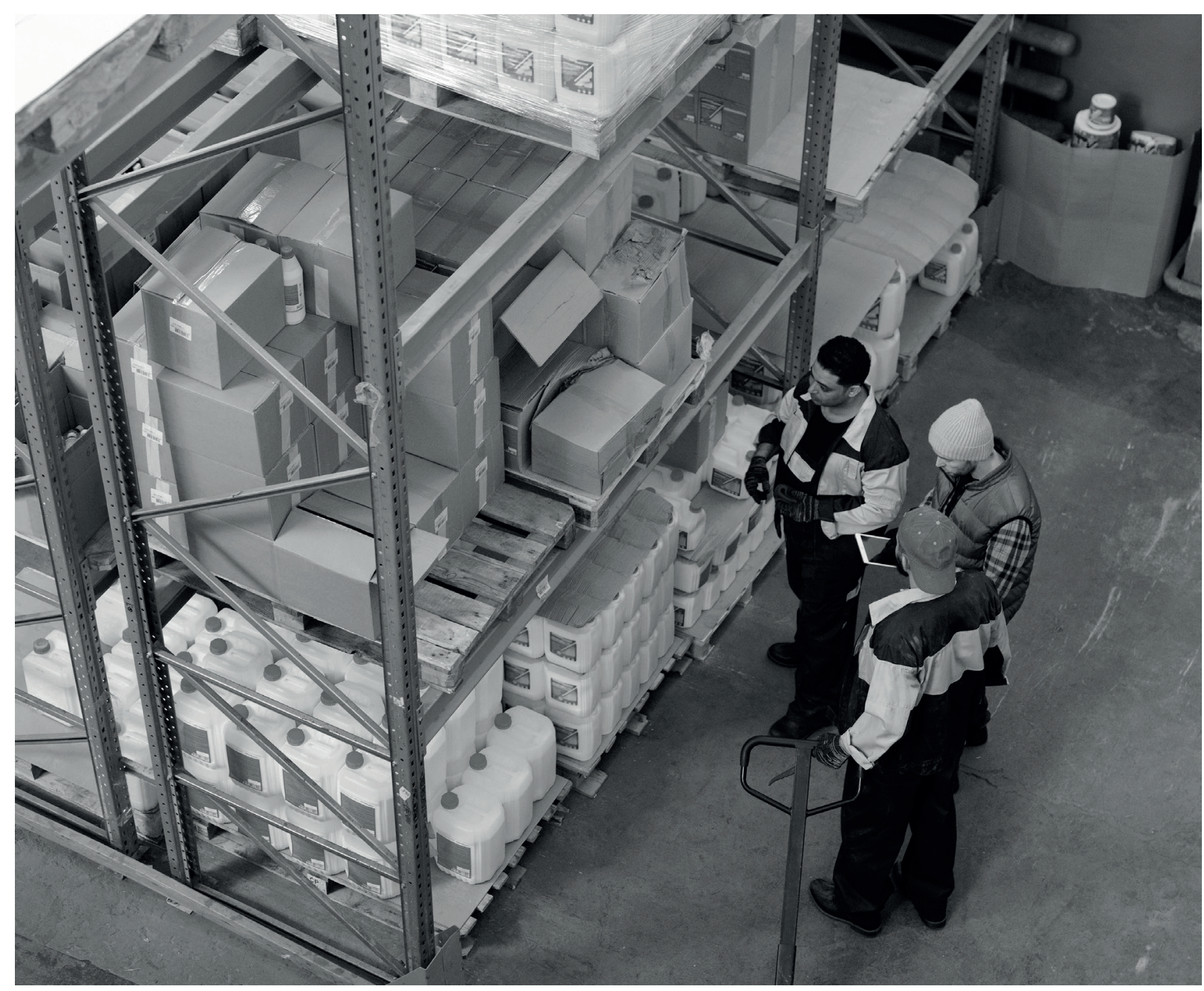

Fuente: Pexels.com Licencia Creative Commons 


\title{
"Planeando las compras en PartesYa": una actividad lúdica para estudiar el MRP ${ }^{1}$
}

\author{
"Planeando las compras en PartesYa": a hands-on activity to \\ study the MRP
}

\author{
Carolina Saavedra-Moreno ${ }^{2}$, Nestor Fabian Ramos Quintero
}

Artículo recibido en abril 30 de 2020; artículo aceptado en julio 22 de 2020

\begin{abstract}
Este artículo puede compartirse bajo la Licencia Creative Commons Atribución-NoComercial-Compartirlgual 4.0 Internacional y se referencia usando el siguiente formato: Saavedra-Moreno, C. y Ramos, N. F. (2021). "Planeando las compras en PartesYa”: una actividad lúdica para estudiar el MRP. I+D Revista de Investigaciones, 16(1), 53-61. http://dx.doi.org/10.33304/revinv.v16n1-2021005.
\end{abstract}

\begin{abstract}
Resumen
Este artículo describe una propuesta de actividad lúdica diseñada para el estudio de la planificación de los requerimientos de materiales (MRP, por sus siglas en inglés), en el curso de Administración de Operaciones, del Programa de Ingeniería Industrial, en una universidad colombiana. Esta temática fue categorizada como un tópico que requería de manera prioritaria el diseño de actividades lúdicas, al estudiar la percepción de los estudiantes en las variables de grado de motivación y grado de dificultad. La lúdica simula el proceso de compra a corto plazo de las materias primas requeridas para la fabricación del producto estrella de una compañía de maquinaria industrial. Cada equipo debe determinar las cantidades a comprar, de tal manera que se minimice el costo total de manejo de inventarios. La actividad vincula información relacionada con la planificación de la producción y el comportamiento de la demanda del producto estrella, así como la capacidad de fabricación del proveedor, los costos de compra de las materias primas y descuentos por compras anticipadas. Esta aplicación práctica busca representar un proceso de compras en un contexto real, con el fin de que los estudiantes puedan comprender la utilidad y aplicación del MRP para el desempeño operativo del sistema de producción.
\end{abstract}

Palabras clave: Aprendizaje activo, lúdica, planeación, requerimientos de materiales.

\begin{abstract}
This paper describes a playful activity designed for studying material requirements planning (MRP) in the course of Administración de Operaciones at an Industrial Engineering program in a Colombian university. The perception of students in the variables of motivation degree and difficulty degree of topics was studied, and MRP was categorized like a priority theme that required a playful activity. The game simulates a short-term purchase process of raw materials of the most important product of an industrial machinery company. Each team should determine the quantities to buy, so that the total cost of inventory management is minimized. The activity includes information related to production planning and demand behavior of the product, the supplier's manufacturing capacity, raw material purchase costs and discounts for anticipated purchases. This practical application represents a purchasing process in a real context.
\end{abstract}

\footnotetext{
${ }^{1}$ Artículo de investigación, de enfoque mixto, resultado de un trabajo de grado de pregrado titulado"Propuesta de actividades lúdicas para el apoyo de los procesos de enseñanza-aprendizaje de la asignatura Administración de Operaciones II del programa de Ingeniería industrial", perteneciente al área de ciencias sociales e ingenierías, subárea de operaciones y procesos, desarrollado en el Grupo de Investigación GINNOVA, programa de Ingeniería Industrial, Universidad de Ibagué (Colombia). Dirección: carrera 22 calle 67, Ibagué, 730002 PBX: +57 (8) 2760010 . Fecha de inicio: julio de 2019. Fecha de finalización: febrero de 2020.

2 Magíster en Gestión Industrial, Universidad de Ibagué. Vinculada al Grupo de Investigación GINNOVA, Programa de Ingeniería Industrial, Universidad de Ibagué (Colombia). Dirección: carrera 22 calle 67, Ibagué, 730002 PBX: +57 (8) 2760010 . ORCID ID: https://orcid.org/0000-00026569-7932. Correo electrónico institucional: carolina.saavedra@unibague.edu.co.

${ }^{3}$ Estudiante de Ingeniería Industrial, Universidad de Ibagué (Colombia). Dirección: carrera 22 calle 67, Ibagué - 730002 PBX: +57 (8) 2760010.0 ORCID ID: https://orcid.org/0000-0003-4913-9102. Correo electrónico institucional: 2320171105@estudiantesunibague.edu.co.
} 
Carolina Saavedra-Moreno, Néstor Fabián Ramos Quintero "Planeando las compras en PartesYa": una actividad lúdica para estudiar el MRP

The objective is that students can understand the utility of MRP for the operational performance of the production system.

Keywords: active learning, playful activity, planning, material requirements.

\section{Introducción}

La ingeniería industrial es multidisciplinar, práctica e interdisciplinaria por naturaleza. Por lo anterior, en los procesos de enseñanza una parte limitada de la teoría puede ser abordada solamente con la metodología de clase magistral de manera efectiva (Lantada et al. 2016). Simpson (2003) resalta que las nuevas tendencias de enseñanza-aprendizaje han abierto un camino en la búsqueda de alternativas que permitan generar un conocimiento activo y aplicable. Involucrar a los estudiantes de manera activa en su aprendizaje les permite generar conocimiento significativo e incrementar el interés en los temas estudiados.

Por lo anterior, en la literatura existe gran diversidad de actividades lúdicas para temáticas de Ingeniería Industrial. Por ejemplo, Polito et al. (2004) proponen una lúdica en la que los estudiantes diseñan el proceso de producción y posteriormente evalúan su desempeño. Por otro lado, Ruiz et al. (2018) y Paredes-Rodríguez et al. (2016) retan a los estudiantes a tomar decisiones claves vinculadas con la gestión de los inventarios, tales como cuánto y cuándo pedir, con el fin de analizar las consecuencias de estas decisiones y afianzar de manera activa los conocimientos del aula de clase.

Teniendo en cuenta que en la asignatura de Administración de Operaciones del programa de Ingeniería Industrial de una universidad colombiana se identificó que se tenía un alto componente de clases magistrales, baja motivación por parte de los estudiantes y una tasa de desaprobación de la asignatura cercana al $10 \%$, se decidió diseñar actividades lúdicas para los temas prioritarios del curso (Acosta-Medina et al., 2020). El objetivo de estas actividades fue disminuir la percepción de dificultad de las temáticas, aumentar el grado de interés en las actividades que se llevan a cabo en el aula y mejorar los resultados académicos de los estudiantes.

Una de las temáticas priorizadas fue la planeación de requerimientos de materiales (MRP), conocida como un sistema de planeación de la manufactura para productos de demanda dependiente, que permite desplegar un conjunto de órdenes de materiales y componentes, con el fin de minimizar inventarios y reducir costos (Chase et al., 2006) (Collier y Evans, 2007). Este sistema es útil para escenarios de manufactura de múltiples etapas, en donde las necesidades de compra de las materias primas dependen del Plan Maestro de Producción (MPS). En este contexto la demanda es discontinua y discreta; el problema es determinar las cantidades de componentes a fabricar y/o comprar y programarlos en las fechas adecuadas, para mantener el flujo de materiales en el sistema (Russell y Taylor, 2017).

En la literatura existen lúdicas diseñadas para abordar la temática MRP entre las que se resaltan la propuesta por Barcante et al. (2011), quienes plantean un juego para estudiar las decisiones de la planificación de la producción que va desde el diseño del producto hasta la planificación de requerimientos de materias primas. A partir de un plan de producción semanal, los equipos deben determinar las cantidades a pedir, el momento de las compras y seleccionar los proveedores, teniendo en cuenta que el objetivo es reducir el costo de fabricación del producto. Durante el juego el facilitador presenta circunstancias inusuales asociadas al proceso de abastecimiento, tales como cancelación de pedidos, aumento de la demanda y retraso en la entrega de materias primas. Teniendo en cuenta todos los sucesos presentados, al final cada equipo debe presentar una propuesta de MRP capaz de cumplir el plan de producción entregado al inicio de la actividad.

Por otro lado, Osorio y Jaramillo (2006) crearon una lúdica denominada "Rouge River y las Mudas", que tiene por objetivos la enseñanza de los conceptos básicos del sistema MRP y la identificación de mudas en el sistema productivo. En la actividad se simula una planta de producción Flow Shop con 6 estaciones de trabajo (de diferentes niveles: materia prima y subensambles) y un área de producto terminado, en donde se fabrican dos tipos de producto. La simulación inicia con la generación de una orden de producción cada minuto. A medida que se desarrolla la lúdica, el gerente general ilustra a los participantes sobre los principales componentes del MRP I, nombra las mudas más comunes e invita a los participantes a que identifiquen dichas pérdidas en el proceso de producción de los productos. Después de la lúdica los estudiantes deben proponer un MRP para cada producto, usando diferentes técnicas de dimensionamiento de lote.

En este artículo se presenta una actividad lúdica para estudiar el MRP. Inicialmente se muestra el proceso metodológico llevado a cabo para la selección de esta temática como uno de los temas críticos de intervención con actividades lúdicas, en el curso de Administración de Operaciones. Posteriormente se detalla la estructura y desarrollo de la actividad. A diferencia de las lúdicas anteriores, en esta propuesta, además de planificar las cantidades a pedir de las materias primas definidas a partir del estudio del plan maestro de producción, se evalúa el desempeño de la planificación al simular los procesos de compra (a un proveedor con restricciones de capacidad 
Carolina Saavedra-Moreno, Néstor Fabián Ramos Quintero

"Planeando las compras en PartesYa": una actividad lúdica para estudiar el MRP

y quien ofrece la posibilidad de tener descuentos por realizar pedidos anticipados) y los procesos de fabricación y venta del producto final, en un escenario de demanda aleatoria periodo a periodo. En el contexto anterior, durante el desarrollo del juego los equipos tendrán la posibilidad de analizar las implicaciones de los tamaños de lote definidos y ajustar la planificación de los requerimientos de las materias primas, teniendo en cuenta su desempeño en cada periodo. Durante el juego se cuenta con un presupuesto establecido para cubrir los costos de manejo de inventarios (compra, ordenar, mantener y faltantes), y se deberá decidir las cantidades de materias primas a comprar, de tal manera que se minimicen los costos totales de manejo de inventarios.

\section{Marco referencial}

El MRP es un sistema de planificación de componentes de fabricación que, mediante un conjunto de procedimientos lógicamente relacionados, traduce el Plan Maestro de Producción (MPS) en necesidades reales de componentes, con fechas y cantidades (Dominguez et al., 1995). La lógica del sistema MRP genera un efecto de demanda dependiente por niveles; es decir, el nivel cero (nivel de producto final) define la programación del nivel uno (subensambles), y así sucesivamente hasta cubrir todos los niveles definidos en el proceso de ensamble. Esta dinámica explica el nombre de productos con demanda dependiente (Krajewski y Ritzman, 2020).

Los principales insumos para realizar un MRP son el MPS o plan detallado, que establece cuántos productos finales serán producidos y en qué periodos de tiempo, la lista de materiales que vincula las cantidades necesarias de cada ítem para fabricar el producto final y la secuencia en que los distintos componentes se combinan para obtener el artículo final, los registros de inventario y compra que presentan la información consolidada de disponibilidad de producto y los plazos de entrega para cada artículo (ver Figura 1) (Schroeder, 2004). Una vez se cuenta con esta información, tomando en consideración la estructura del producto, las existencias actuales y los compromisos de entrega, se elabora un listado de materiales para satisfacer el MPS. Este listado muestra cuándo se debe pedir un artículo a los proveedores, si hay inventario disponible y cuándo la producción de un artículo debe comenzar para satisfacer la demanda del producto terminado en una fecha particular (Render y Heizer, 2014).

Al comparar el sistema MRP con los modelos de gestión de inventarios para demanda independiente, se identifican como ventajas la posibilidad de adaptarse a las necesidades del MPS, con sus variaciones de demanda, las cuales pueden ser transmitidas a los diferentes niveles del proceso y/o a los proveedores. Otra ventaja es la capacidad para ordenar lotes de diversos componentes de forma simultánea y coordinada, teniendo en cuenta los niveles de existencia y los compromisos de despacho (Saracche y Morales, 2016).

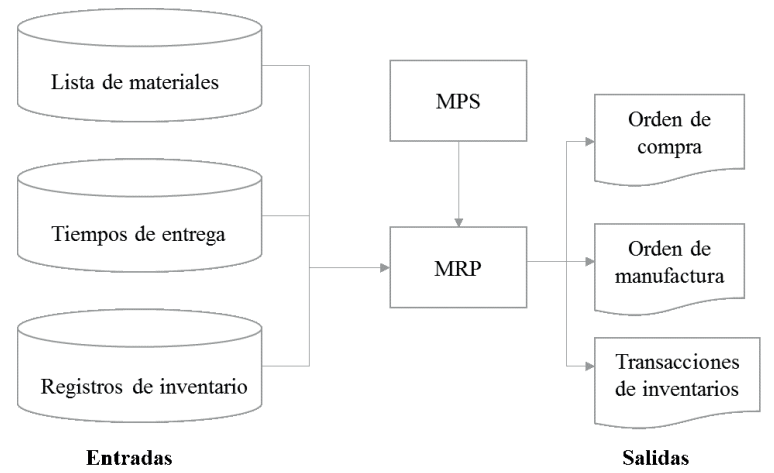

Figura 1. Estructura MRP. Fuente: Adaptado de Render y Heizer (2014).

\section{Metodología}

Para definir para qué temáticas del curso se debían diseñar actividades lúdicas, se realizó un proceso de priorización de tópicos, que se desarrolló a partir del estudio de las variables grado de dificultad y grado de motivación de los estudiantes. Se aplicó una encuesta por medio de Google Forms a 18 estudiantes que ya habían tomado la asignatura y a 3 docentes del programa de Ingeniería Industrial que dictan o han dictado la asignatura. Esta encuesta se estructuró en dos partes. En la primera los encuestados debían calificar de manera independiente, de 1 a 5, el grado de dificultad y el grado de motivación que percibían de cada uno de los temas. Como segunda medida, por medio de preguntas abiertas, se consultó respecto a las razones de las calificaciones anteriormente dadas y las estrategias que los encuestados consideraban válidas para disminuir el grado de dificultad e incrementar la motivación.

Se diseñó una matriz de priorización (ver Figura 2), en donde, a partir de la relación entre el grado de dificultad y grado de interés, se clasifica cada tema en alguno de los siguientes cuatro cuadrantes: éxito (temas de alto interés y bajo nivel de dificultad - cuadrante ideal), motivación (temas de bajo interés y bajo nivel de dificultad), prioridad (temas de bajo interés y alto nivel de dificultad) y crítico (temas de alto interés y alto nivel de dificultad). Dentro de la matriz, se ubicaron las temáticas según la calificación promedio obtenida en las dos categorías de evaluación, y a partir de esta categorización se seleccionó la temática que requería de una intervención prioritaria.

En la siguiente fase se determinó la estructura de la actividad lúdica para el tema priorizado, teniendo en cuenta los principios de diseño de juegos recopilados por Contreras y Eguia (2017). A continuación se presentan los elementos constitutivos de la estructura de la lúdica:

- Información general: asignatura, tema y objetivos de aprendizaje.

- Narrativa: se define el contexto empresarial en el 
Carolina Saavedra-Moreno, Néstor Fabián Ramos Quintero "Planeando las compras en PartesYa": una actividad lúdica para estudiar el MRP

que se desarrolla la actividad, los roles que cada uno de los asistentes debe cumplir, las estaciones de trabajo, el objetivo de la lúdica, datos de entrada, las bonificaciones y las instrucciones del desarrollo de la actividad.

- Recursos y tiempo: materiales, formatos y duración estimada.

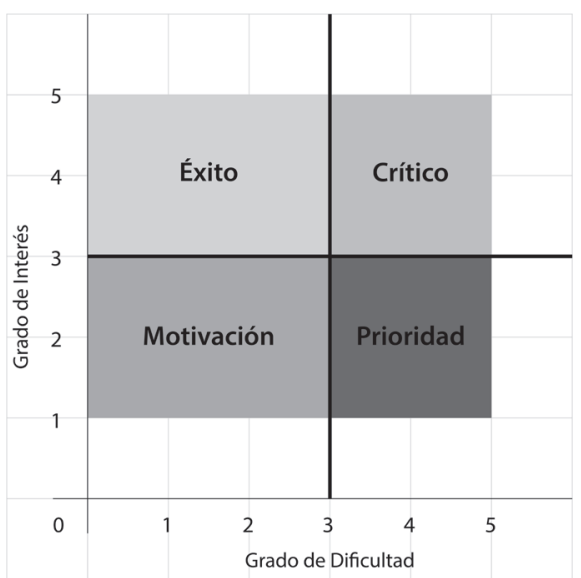

Figura 2. Matriz de calificación. Fuente: Autores.

Finalmente se llevó a cabo una validación preliminar con un grupo de 9 participantes entre estudiantes y profesores, quienes realizaron la actividad propuesta, durante un periodo de 2 horas, en el laboratorio del programa de Ingeniería Industrial (ver Figura 3). En particular participaron siete estudiantes de sexto/ séptimo semestre del programa de Ingeniería Industrial, un estudiante de octavo semestre de Ingeniería de Sistemas y un docente del programa de Ingeniería Industrial.

Al finalizar la sesión se realizó una socialización de la experiencia con los participantes, en donde se indagó por la claridad de la información registrada en los formatos, la facilidad para realizar la lúdica de manera autónoma y el grado de motivación a la hora de ejecutarla. Los participantes compartieron de manera oral sus percepciones al respecto.

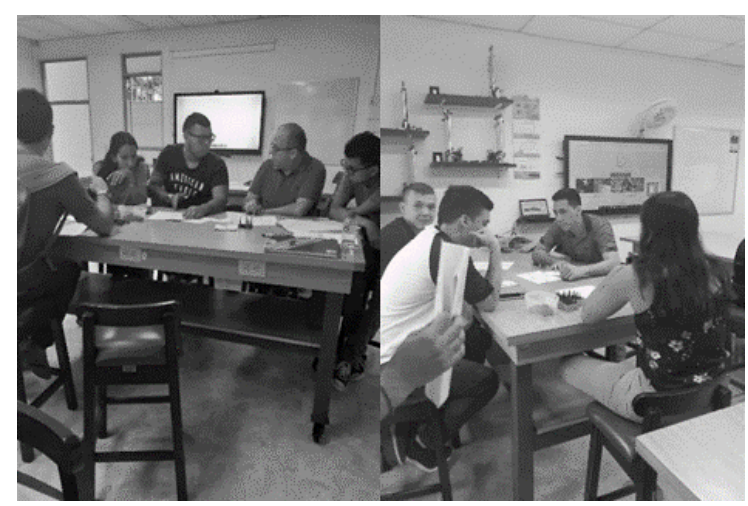

Figura 3. Registro fotográfico validación preliminar. Fuente: Autores.

\section{Resultados}

A continuación se presentan los resultados obtenidos en la priorización de las temáticas y el diseño completo de la lúdica con los resultados de su validación preliminar

\section{Fase 1. Priorización de temáticas}

En la Figura 4 se muestra la ubicación de las temáticas de la asignatura en la matriz de priorización. Se observa que tanto en el cuadrante de motivación como en el de prioridad no se ubica ninguna de las temáticas del curso. Se encontró que todos los promedios de las variables grado de interés y grado de dificultad eran superiores a 3. En el cuadrante crítico se ubicaron las temáticas: Plan Agregado de Producción, el Plan Maestro de Producción y la Planeación de Requerimientos de Materiales (MRP). Los demás temas se ubicaron en el cuadrante "Éxito". De las temáticas que se encuentran en el cuadrante 3 , MRP fue la que presentó el nivel promedio más alto de dificultad y el valor medio más bajo de interés. Por lo anterior, esta temática fue elegida como el tópico para diseñar la actividad lúdica.

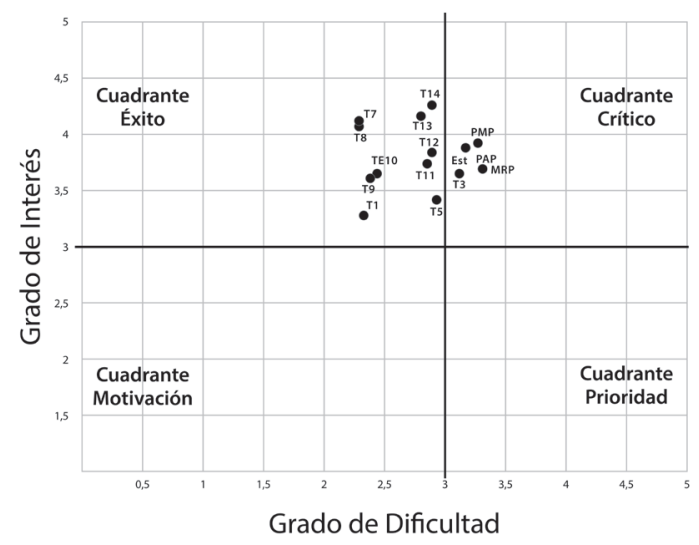

Figura 4. Priorización de las temáticas. Fuente: Autores.

\section{Fase 2. Diseño de la lúdica "Planeando Las Compras En PartesYa"}

\section{Información general}

- Asignatura: Administración de Operaciones

- Tema: Planeación de Requerimientos de Materiales (MRP)

- Objetivos de aprendizaje:

- Identificar la importancia de planificar los requerimientos de materiales en el desempeño organizacional

- Analizar el desempeño en costo de la utilización de diferentes tamaños de lote en el proceso de planificación de los requerimientos de materiales, teniendo en cuenta condiciones de oferta y demanda 
Carolina Saavedra-Moreno, Néstor Fabián Ramos Quintero

"Planeando las compras en PartesYa": una actividad lúdica para estudiar el MRP

\section{Narrativa}

- Contexto: La actividad se construye para la empresa PartesYa, una reconocida compañía colombiana que produce partes para maquinaría industrial de tipo eléctrico e hidráulico. El producto estrella de la empresa es el SKU X340, una bomba volumétrica que hace parte del grupo de maquinaria hidráulica (ver Figura 5).
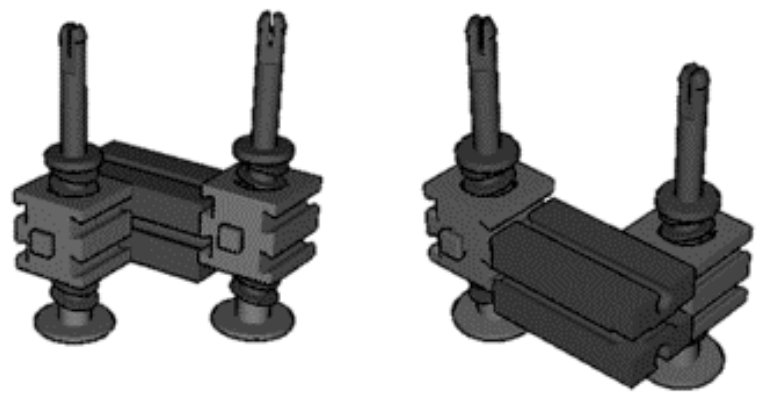

Figura 5. Referencia X340. Fuente: Autores.

- Roles: Un proveedor y 3 fabricantes

El estudiante que cumple el rol de proveedor recibe y revisa las órdenes de compra cada semana. Además, entrega las partes encargadas por la compañía en el periodo definido.

Los tres estudiantes fabricantes ensamblan las unidades cada vez que reciben materias primas. Abastecen el mercado dependiendo de la demanda y generan las órdenes de compra a partir de la planificación de los requerimientos de materias primas que realizan.

- Estaciones de trabajo: Se definen dos estaciones de trabajo interconectadas (proveedor-fábrica).

- Objetivo de la lúdica: planificar los requerimientos de materias primas del producto X340, de tal manera que se minimicen los costos totales de manejo de inventarios.

Al finalizar la actividad se espera que cada equipo identifique el método o técnica de dimensionamiento de lote a pedir, que mejor se ajusta a cada tipo de material, con el fin de minimizar los costos de manejo de inventarios, teniendo en cuenta las condiciones de oferta y demanda vivenciadas.

- Datos de entrada:

- Plan maestro de producción actual

- Demanda: la demanda semanal fluctúa entre 0-5 unidades (se determina con un dado al inicio de cada periodo)
- Lista de materiales y estructura de ensamble de la referencia X340

- Precio de venta de las materias primas

- Tiempo de entrega de las materias primas

- Capacidad de abastecimiento del proveedor

- Tiempo de fabricación de la referencia X340

- Descuentos por compras anticipadas

- MRP actual (el que usa la empresa): para la primera semana del juego se usa la información del MRP actual para definir las cantidades a comprar.

- Posibilidad para incrementar capacidad de fabricación a corto plazo: condiciones de subcontratación de unidades (capacidad y costo).

- Costos de manejo de inventarios (compra, mantener, ordenar y faltantes).

- Presupuesto para cubrir costos de manejo de inventarios.

- Bonificaciones: en la actividad se han definido dos tipos de bonificaciones. La primera se otorga al terminar el primer y tercer periodo. Los equipos que presenten los menores costos acumulados de manejo de inventarios pueden conocer anticipadamente la demanda del siguiente periodo, con el fin de que puedan vivenciar la importancia de una adecuada estimación de demanda. Para incentivar la utilización de técnicas de dimensionamiento de lote aprendidas en el curso, se tiene definida una segunda bonificación en dinero, para los equipos que en su proceso de planificación de requerimientos de material utilicen las técnicas teóricas abordadas.

- Instrucciones generales: para cada semana se llevan a cabo las siguientes fases:

a) Cada equipo define la estrategia de planificación de materiales que va a utilizar, teniendo en cuenta la información de entrada disponible y la experiencia en el juego. A partir de la estrategia definida el equipo genera o no una orden de compra.

b) El proveedor revisa las órdenes de compra y envía las partes que se solicitaron.

c) Los fabricantes ensamblan las unidades con las materias primas recibidas.

d) Se conoce la demanda del periodo y se procede a suplirla con las unidades disponibles. Si no se cuenta con las unidades requeridas los fabricantes pueden decidir entre subcontratar o perder la venta, con los respectivos costos asociados.

e) Se calculan los costos de manejo de inventarios por periodo.

f) Los equipos con menor costo en la semana 4 y 12 ganarán la bonificación de conocer la demanda de 
forma anticipada. Los demás equipos conocerán la demanda al transcurrir cada uno de los periodos. En estos mismos periodos se entregará la bonificación 2 a los equipos que usaron técnicas de dimensionamiento de lote trabajadas en clase para planificar las compras.

g) Al finalizar se revisarán los costos totales por grupo; el que tenga los menores costos será el equipo ganador.

h) Extraclase: a partir de la vivencia del juego, cada equipo deberá proponer a la empresa la técnica de dimensionamiento de lote que se debe utilizar para pedir cada materia prima, de tal manera que se minimicen los costos de manejo de inventarios, teniendo en cuenta el contexto del proceso de planificación de los materiales.

\section{Recursos y tiempo}

A continuación se presenta el listado de materiales y formatos requeridos por equipo para el desarrollo de la lúdica.

- Para la construcción de una referencia X340 se requieren las piezas de FischerTechnik que se presentan en la Figura 6. Cada equipo requiere 20 unidades de tres de las cuatro fichas y 40 de la restante.
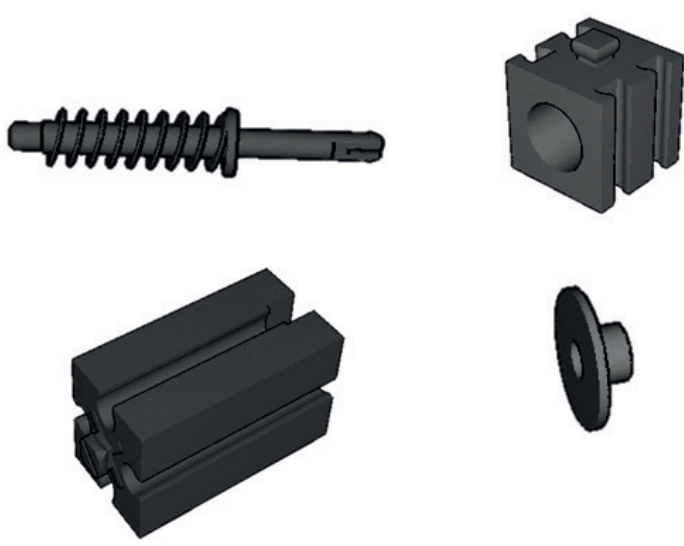

Figura 6. Piezas de FischerTechnik. Fuente: Autores por medio del software Fischertechnick Designer.

- Un dado de seis caras para determinar la demanda

- Formato de orden de compra que se diligencia cada vez que el equipo desea comprar unidades (ver Figura 7)

- Tablero de juego. En el tablero semana a semana se registra la información de demanda, inventario disponible, unidades subcontratadas, ventas perdidas y costo total (ver Figura 8).

La duración estimada de la lúdica es de 2 horas.

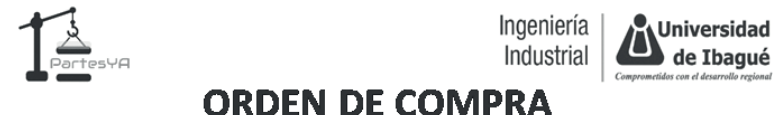

PROVEEDOR:

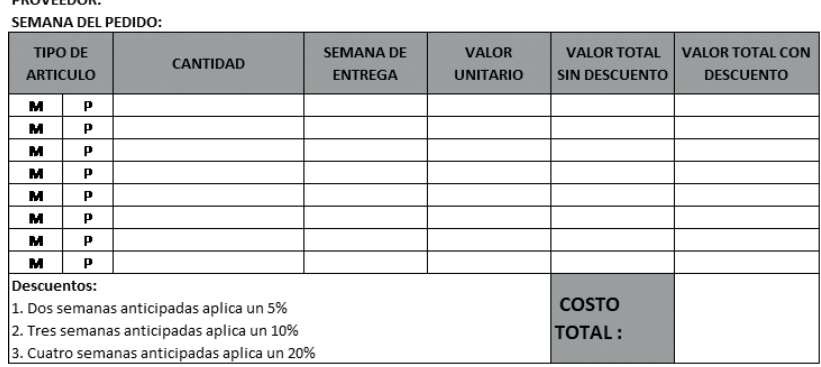

Figura 7. Formato orden de compra. Fuente: Autores.

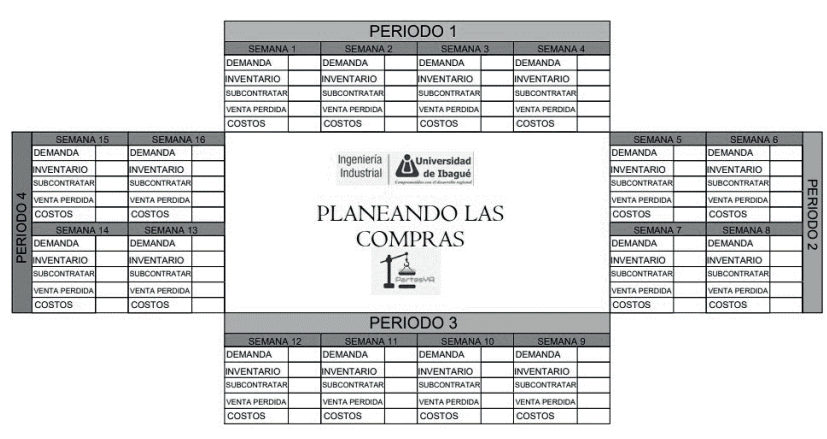

Figura 8. Formato tablero de juego. Fuente: Autores.

\section{Fase 3. Validación preliminar}

Como resultado de la validación, se obtuvo que el $100 \%$ de los participantes consideró que la actividad fue pertinente, interesante, divertida y que se cumplía con el objetivo propuesto.

Con respecto a la claridad de la información registrada en los formatos, dos de los participantes resaltaron la necesidad de que cada equipo tuviera un formato adicional en donde se registren de manera detallada los costos, para facilitar el cálculo del costo total que se registra en el tablero de juego. Además, se recomendaron modificaciones en el formato de compra (particularmente separar el valor total sin descuento y con descuento) con el fin de facilitar el cálculo del costo total (esta recomendación fue incorporada en el formato).

Finalmente, uno de los participantes resaltó que las instrucciones del juego eran claras y permitían el desarrollo autónomo del ejercicio. Además, que es muy importante el papel del docente como motivador, tanto para la obtención de las bonificaciones como para incentivar la competencia entre los equipos.

\section{Conclusiones}

Como primera medida se propone una metodología para priorizar las temáticas a las que se les debe diseñar actividades lúdicas, a partir del estudio de las 
Carolina Saavedra-Moreno, Néstor Fabián Ramos Quintero

"Planeando las compras en PartesYa": una actividad lúdica para estudiar el MRP

variables grado de dificultad y grado de motivación. Esta metodología puede ser utilizada para cualquier asignatura; es una herramienta que permite categorizar los temas en cuatro grupos (éxito, motivación, prioridad y crítico). Lo ideal es que todos los temas estuviesen en el cuadrante de éxito. A partir de la categorización es posible que el docente pueda plantear diversas estrategias para alcanzar este fin. La metodología fue usada para priorizar las temáticas en el curso Administración de Operaciones II del programa de Ingeniería Industrial, de una universidad colombiana y sirvió de base para identificar que la temática prioritaria de intervención era la Planificación de los Requerimientos de Materiales (MRP).

Como segunda medida se presenta una lúdica para estudiar el MRP. En el marco de un contexto empresarial, los equipos de estudiantes deben planificar los requerimientos de materias primas para un producto. Además, realizar el proceso de compra de las materias primas y venta del producto final. Por medio de esta actividad y teniendo en cuenta un conjunto de condiciones de oferta y demanda, los estudiantes comprenden la importancia del MRP para el desempeño organizacional, así como el impacto del tamaño de lote de una orden de compra para el costo de manejo de inventarios.

A diferencia de lúdicas anteriores, esta actividad permite que el estudiante, además de comprender la dependencia entre las decisiones de planificación de las materias primas y la demanda del producto final, pueda evaluar el desempeño en costo de la planificación de materiales propuesta, al simular los procesos de compra de las materias primas y de venta del producto final. Por medio de la realización de esta lúdica los estudiantes pueden vivenciar las interrelaciones entre las decisiones de planificación de la producción y las condiciones de oferta y demanda.

\section{Referencias}

Acosta-Medina, J. K., Torres-Barreto, M. L., ÁlvarezMelgarejo, M. y Paba-Medina, M. C. (2020). Gamificación en el ámbito educativo: Un análisis bibliométrico. I+D Revista de Investigaciones, 15(1), 30-39. https://doi.org/10.33304/revinv.v15n12020003

Barcante, L., Ramos, F. y Seno, C. (2011). The MRP Game: A Decisive Method to Teach Production System. ISAGA 2011 Conference. https://www.researchgate. net/publication/277142237_The_MRP_Game_a_ decisive_method_to_teach_production_system

Chase, R. B., Jacobs, F. R. y Aquilano, N. J. (2006). Operations Management for Competitive Advantage. McGrawHill-Irwin.
Collier, D. A. y Evans, J. R. (2007). Operations Management. Goods, Services and Value Chains. (2. ${ }^{\mathrm{a}}$ ed)Thomson South-Western.

Contreras, R. S. y Eguia, J. L. (2017). Gamificación en escenarios educativos, revisando literatura para aclarar conceptos. In InCom-UAB Publicacions Experiencias de Gamificación en Aulas. 7-17.

Dominguez, J., Garcia, S., Dominguez, M., Ruiz, A. y Alvarez, M. J. (1995). Dirección de operaciones. aspectos tácticos y operativos en la producción y los servicios. McGraw-Hill.

Krajewski, L. J. y Ritzman, L. P. (2020). Administración de operaciones: Estrategia y análisis. (5. ${ }^{\mathrm{a}}$ ed) Pearson Educación.

Lantada, A. D., Muñoz-Guijosa, J. M., Chacón, E., Echávarri, J. y Muñoz, J. L. (2016). Engineering Education for All: Strategies And Challenges. International Journal of Engineering Education, 32(5), 2155-2171.

Osorio, M. y Jaramillo, C. (2006). Utilización de la lúdica para la enseñanza del MRP I. Scientia et Technica, 12(32), 301-306.

Paredes-Rodríguez, A. M., Peláez-Mejía, K. A. y SalazarRamos, A. F. (2016). Propuesta de un juego de mesa como herramienta didáctica para la explicación de conceptos de control de inventarios en programas de ingeniería industrial. Revista Educación en Ingeniería, 11(21), 45-50.

Polito, T., Kros, J. y Watson, K. (2004). Improving Operations Management Concept Recollection Via the Zarco Experiential Learning Activity. Journal of Education for Business, 79(5), 283-286. https://doi. org/10.3200/JOEB.79.5.283-286

Render, B. y Heizer, J. (2014). Principios de administración de operaciones. (9. a ed) Pearson Educació.

Ruiz, C. R., Castiblanco, I. A., Cruz, J. P., Pedraza, L. C. y Londoño, D. (2018). Juegos de simulación en la enseñanza de la Ingeniería Industrial: caso de estudio en la Escuela Colombiana de Ingeniería Julio Garavito. Entre Ciencia e Ingeniería, 12(23), 4857. https://doi.org/10.31908/19098367.3702

Russell, R. S. y Taylor, B. W. (2017). Operations and Supply Chain Management. (9. ${ }^{\text {a }}$ ed) John Wiley y Sons.

Saracche, W. y Morales, M. (2016). Localización, transporte e inventarios. Tres decisiones estructurales en el diseño de cadenas de abastecimiento. Universidad Nacional de Colombia. 
Schroeder, R. G. (2004). Administración de operaciones: Casos y conceptos contemporáneos. ( $\left.2 .^{\mathrm{a}} \mathrm{ed}\right)$. McGraw-Hill.

Simpson, T. W. (2003). Experiences with a Hands-on Activity to Contrast Craft Production and Mass Production in the Classroom. International Journal of Engineering Education, 19(2), 297-304. 